\title{
Corrosion protection for cold-formed structural steel elements
}

Unprotected steel and steel structures exposed to the atmosphere inevitably corrode. For thin-gauged structures especially, corrosion is critical for stability. Although the reduction in thickness caused by corrosion is insignificant for some structures, in other structures corrosion is unacceptable. Therefore, corrosion protection is a crucial issue for steel structures and the steel industry. Over the last decades, many new corrosion protection systems and innovative steel products have been introduced onto the market. Corrosion protection for cold-formed structural steel elements has been standardized through the introduction of the EN 1090-4. This paper introduces the harmonized rules for the corrosion protection of loadbearing thingauged structures. The zinc-magnesium-aluminium (ZM) metal coating system developed in recent years is also dealt with.

Keywords EN 1090-4; corrosion protection; lightweight structures; thin-gauged; zinc-magnesium-aluminium (ZM)

Introduction

Corrosion protection is a crucial issue for steel structures, especially thin-gauged structures. In Europe the protection of thin-gauged steel and strips or products made from this are regulated by a large number of European and national standards. Some of these standards regulate the general options for corrosion protection, while others deal with the concerns of special applications. Over the last decades, many new corrosion protection systems and innovative steel products have been introduced onto the market. Corrosion protection for cold-formed structural steel elements has been standardized through the introduction of the EN 1090-4 [1]. This standard has largely

Tab. 1 Changes to periods of protection in EN ISO 12944-1

\begin{tabular}{lll}
\hline \multicolumn{2}{l}{ Duration of protection } \\
\hline & EN ISO 12944-1:2018 & EN 1090-4:2008 and \\
& & EN ISO 12944-1:1998 \\
\hline Low $(\mathrm{L})$ & $\leq 7$ years & $2-5$ years \\
Medium $(\mathrm{M})$ & $7-15$ years & $5-15$ years \\
High $(\mathrm{H})$ & $15-25$ years & $>15$ years \\
Very High $(\mathrm{VH})$ & $>25$ years & - \\
\hline
\end{tabular}

This is an open access article under the terms of the Creative Commons Attribution-NonCommercial-NoDerivs License, which permits use and distribution in any medium, provided the original work is properly cited, the use is non-commercial and no modifications or adaptations are made. closed the gap in standardization for cold-formed structural steel elements. Unfortunately, some aspects of corrosion protection remain unresolved.

This paper provides an overview of the standards for thingauged steel and introduces the harmonized rules for corrosion protection of thin-gauged structures. The zincmagnesium-aluminium (ZM) metal coating system developed in recent years is also dealt with.

\section{Current state of standards for corrosion protection of thin-gauged cold-formed products}

\section{$2.1 \quad$ General}

EN ISO 12944 forms the basis for the corrosion protection of steel structures. This standard regulates all aspects of corrosion protection, such as surfaces, surface preparation, coating systems, test procedures and, in Part 2, in particular the classification of environmental conditions with the specification of corrosivity categories $C$ and the associated corrosion rates of unalloyed steel and zinc. In 2018 the definitions of the protection periods were changed in EN ISO 12944-1 [2] and the definitions of the corrosion categories C in EN ISO 12944-2 [3]. That means that now neither matches the regulations in EN 1090-4 [1], the standard that covers corrosion protection for cold-formed structural steel elements, which is still based on the previous versions. Tab. 1 and Tab. 2 show the differences between the two versions.

EN ISO 12944-5 [4] specifies the coating systems for use in the corrosivity categories according to EN ISO

Tab. 2 Changes to corrosivity categories in EN ISO 12944-2

\begin{tabular}{ll}
\hline Corrosivity categories \\
\hline EN ISO 12944-2:2018 & EN \\
\hline C1 very low \\
C2 low \\
C3 medium \\
C4 high
\end{tabular}

C5 very high $\quad$ C5-I very high (industrial)

CX extreme 
12944-2 and the duration of protection according to EN ISO 12944-1. For cold-formed products, especially for thin gauges, EN ISO 12944-5 is of minor importance. The products with continuously hot-dip coated metal coatings and organic coil coatings play a greater role here. EN 10346 [5] specifies the requirements for continuously hot-dip coated flat steel products. This standard covers all applications of hot-dip coated steel sheets, such as for the automotive and domestic appliances industries, and is not limited to the construction sector. Therefore, metal coatings that are not used for structural products are also included in this standard. EN 10346 does not stipulate any corrosion performance for the metal coatings. The additional organic coil coating is regulated by EN 10169 [6], which specifies the requirements for continuously organically coated flat steel products, primarily because of the service properties of the coatings. This standard covers all applications of organically coated steel sheet, such as for the automotive and domestic appliances industries, and is not limited to the construction sector. This must be taken into account when selecting coating systems. The classification of the coating systems for a corrosivity category may be based on short-term tests or outdoor exposure tests, although the outdoor exposure with a duration of two years is very short compared with the required design working life. In addition, the permissible damage to the organic coating after two years of outdoor exposure is relatively large. Therefore, the classification of the coating system with regard to the corrosivity category in this standard provides information about general suitability but does not provide any information about the suitability for construction products. Furthermore, the properties of the coating system (exept coating thickness and dimensions) are only guaranteed if the purchaser orders them. Therefore according to the section 5 in EN 10169 the purchaser has to specify all the properties of the coated material he needs and even the tests which has to be done in the factory production control to ensure these properties. Also the classification tests with regard to the corrosivity category shall be specified by the purchaser. In practice, this is certainly not feasible, as the specialist competence here lies with the manufacturer.

Corrosion protection for cold-formed structural steel members and sheeting (construction product), especially for roof, ceiling, floor, wall and cladding applications, is covered by EN 1090-4 [1]. This standard was published in 2018 and contains an annex E "Corrosion protection by metallic coating with or without organic coatings". EN 1090-4 contains application rules for construction products, but application rules for corrosion protection are not listed. However, when selecting construction-specific corrosion protection in particular, the planner is faced with questions regarding the appropriate corrosion protection systems. This gap is not part of the standardization route and has to be filled by additional technical rules, such as the international or national technical rules of professional associations.
Another harmonized standard for steel sheeting exists alongside EN 1090-4 [1] for structural applications: EN 14782 [7] is the product standard for self-supporting metal sheet for roofing, external cladding and internal lining. This and the associated non-harmonized standard EN 508-1 [8] both contain some information on corrosion protection.

\subsection{Corrosion protection regulations in European construction product standards}

\subsubsection{Self-supporting products}

For self-supporting metal sheet, EN 508-1 [8] contains a list of materials that are suitable for such applications. The current version covers steel with metal coatings in the form of zinc $(\mathrm{Z})$, zinc-aluminium (ZA, $5 \% \mathrm{Al}-\mathrm{Zn})$, aluminium-zinc (AZ, $55 \% \mathrm{Al}-\mathrm{Zn}$ ) and aluminium (A). The new zinc-magnesium-aluminium $(\mathrm{ZM}, \mathrm{Al}$ and $\mathrm{Mg}$ compositions off $1.5-8 \%$ where the magnesium content is at least $0.2 \%$ ) coatings are not yet included but will be with the next revision, which might be ready in 2021. A comprehensive list of organic coatings helps the user decide which types of coating are available for such products. Annex C "Metal coatings" includes informative tables that show which national requirements exist in many (not all) European countries regarding the minimum nominal thicknesses of metal coatings depending on the application. There are tables for the minimum nominal metallic coating mass for steel sheets depending on the application, indoor or outdoor, and the presence of an organic coating.

As mentioned before, annex C of EN 508-1 [8] is only an informative annex. Therefore, the data given do not represent requirements but only show the existing national regulations, which should be reviewed as they may change in the meantime and may vary depending on the final application of the product.

EN 508-1 is a non-harmonized product standard. The harmonization (CE marking) is regulated by the harmonized umbrella standard EN 14782 [7], which not only covers the aforementioned steel products, but also other metals. In this standard, corrosion protection is covered by the section "Durability". No requirement is given regarding tests; only the type and thickness (or mass) and/ or category of any coating(s) have to be stated. This information should also be part of the CE marking or the Declaration of Performance (DoP).

EN 14782, in combination with EN 508-1, does not contain any requirements regarding testing or Factory Production Control (FPC) of the corrosion protection system. The manufacturers only need to verify, that the inspection document of the suppliers in accordance with EN 10204 [9] indicates, that the base materials (e.g. coils, sheets) have the characteristics that they need to manufacture the construction product. Both standards only 
enable users to select products that may be expected to provide the required product durability with regard to the expected environment and/or exposure conditions and feasibility of maintenance.

\subsubsection{Structural products}

EN 1090-4 [1] regulates primarily the manufacture of cold-formed structural steel elements and the installation of cold-formed structures for roof, ceiling, floor, wall and cladding applications. The scope of the standard does not specify any thickness limitation for the application of the given rules. Therefore, the regulations given in annex $\mathrm{E}$ are not limited to any material thickness; any limitation is due to the cold-forming production process, and a limitation such as "thin-gauged" is not given. Other topics such as material selection, maintenance and surface protection are also dealt with in the standard. This standard represents an attempt to regulate the entirety of all questions for loadbearing thinwalled structures. Before the publication of this standard, the rules of application for structural cold-formed elements in Europe were partly very different and pursued different concepts. The standardization committee faced the big challenge of bringing all these national differences to a consensus. As already mentioned, for thinwalled elements especially, corrosion protection cannot be separated from construction. For the adequate design of structures in terms of corrosion protection, the consensus was less successful.

In addition to DIN EN 10169 [6], which only considers organic coil coating, EN 1090-4 [1], annex E, evaluates the corrosion protection system of all the layers of metal coatings and/or organic coating materials. Test methods and evaluation criteria are specified for this. EN 1090-4, annex $\mathrm{E}$, is much more detailed than the regulations for self-supporting products. It contains information on how to perform tests and gives measurement provisions for the initial inspection of corrosion protection systems to evaluate the general suitability of a system. Provisions for type-testing and FPC complement these regulations.

For the end-user, Tables E.5 and E. 6 are among the most important parts of annex E. Here, different common organic coating systems on metal coatings are assigned to corrosivity categories and durations of protection. These classifications are only examples to enable the user to make a preliminary selection. The final assessment of suitability for the respective application should always be made by the manufacturer of the construction products.

The special feature of duplex systems, consisting of metal coating and organic coating, is that, due to the special form of interaction between the two components, the ensuing duration of protection is greater than that achieved by just adding together the separate durations of protection of the organic coating and the metal coating. The stated protection duration is based on experience and may vary for different coating system manufacturers. The tables in annex E represent a common consensus across all coating system manufacturers. It is therefore possible that individual manufacturers may also specify other, usually higher, durations for certain organic coating systems.

For the end-user, the second important part of annex E are Tables E.2, E.3 and E.4. These tables contain important information for lightweight metal construction for determining the required corrosion protection classes for wall systems (Tab. E.2), roof systems (Tab. E.3) and floor and ceiling systems (Tab. E.4).

3

\section{Current design requirements for durability}

A decision of the European Commission in 1975 for a programme of action in the field of construction, and related decisions, laid down the basic requirements for construction works and construction products. The decision states: "Construction works as a whole and in their separate parts must be fit for their intended use, taking into account in particular the health and safety of persons involved throughout the life cycle of the works. Subject to normal maintenance, construction works must satisfy these basic requirements for construction works for an economically reasonable working life."

It were these decisions that led to the Eurocode programme being established. Eurocode 0 (EC0, Standard EN 1990 [10]) describes principles and requirements for the safety, serviceability and durability of structures. The Eurocodes follow the same principles. EC0 lays down fundamental requirements for the durability of structures, with the principal requirement for durability defined in section 2.4. According to EC0, a structure shall be designed such that deterioration during its design working life does not impair the performance of the structure taking into account its environment and the anticipated scope of maintenance. In order to achieve an adequately durable structure, key points, e.g. expected environmental conditions, protective measures and intended maintenance during the design working life, should be taken into account. The appropriate requirements for design are defined for steel structures in EC3 (standard series EN 1993).

The design working life is defined in Tab. 2.1 in section 2.3 in EC0 [10] and is illustrated in Tab. 3.

EN 1993-1-1 (EC3) [11] defines the requirements for the durability design of buildings and their components for environmental actions in section 2.1.3.3 and chapter 4 . The required durability can be achieved by choosing an appropriate material or by choosing an appropriate corrosion protection system. Parts susceptible to corrosion should be designed such that inspection, maintenance and reconstruction can be carried out satisfactorily and that access is available for in-service inspections and maintenance. Suitable permanent corrosion protection 
Tab. 3 Design working life of buildings

\begin{tabular}{lll}
\hline $\begin{array}{l}\text { Design working } \\
\text { life category }\end{array}$ & $\begin{array}{l}\text { Indicative design } \\
\text { working life [years] }\end{array}$ & Examples \\
\hline 1 & 10 & Temporary structures $^{(1)}$ \\
2 & 10 to 25 & Replaceable structural parts, e.g. gantry girders, bearings \\
3 & 15 to 30 & Agricultural and similar structures \\
4 & 50 & Building structures and other common structures \\
5 & 100 & Monumental building structures, bridges, and other civil engineering structures \\
\hline
\end{tabular}

(1) Structures or parts of structures that can be dismantled with a view to being reused should not be considered as temporary.

measures must be taken for components that cannot be inspected. For internal building structures, EC3 does not require corrosion protection if the internal relative humidity does not exceed $80 \%$.

However, the requirements set down in EC3 are general and give neither information about the design procedure nor specific requirements for the corrosion protection. That information is provided by further standards for corrosion protection or by standards for construction products. An overview of these standards was presented in the previous section.

To fulfil the requirements of EC0 [10] and EC3 [11], the designer should take into account the corrosive load consisting of the atmospheric conditions and the conditions at the location of the building as well as the necessary durability and safety level of the structure. For thin-walled structures especially, the effectiveness of the corrosion protection also depends on structural details. Therefore, the structural details also have to be considered in the design of a building. A building should be designed in such a way that it reaches its expected lifetime. This can be done by choosing a durable material and/or by corrosion protection. The durability of the corrosion protection often does not equal the lifetime of the building and the protection will require maintenance. The designer should be aware that the selection of the corrosion protection system and/or the material has consequences for the lifetime and/or maintenance costs of the structure.

Publication of the EN 1090-4 [1] standard means that a regulation for cold-formed structural steel elements is now available. This standard regulates the product from material selection to its use in a building. The requirements and the application of the standard with regard to corrosion protection and appropriate design are covered in the next section.

\section{Corrosion protection according to EN 1090-4}

\subsection{General}

In chapter 5 "Constituent products" of EN 1090-4 [1], the surface protection is dealt with in section 5.9. For corro- sion protection systems, there is a reference to chapter 10 and annex E in the standard. For material selection for stainless steel, the standard refers to EN 1993-1-4 [12] (stainless steels are not dealt with any further in this article).

For durability requirements a note is included: "Buildings are normally designed for duration high ' $\mathrm{H}$ ' (EN ISO 12944-1 [2]) if not otherwise specified." This note contradicts the requirements of Eurocode 0, where for most the buildings a design working life of 50 years is appropriate. Actually, this remark should state that, in principle, corrosion protection systems should be used which provide at least the protection duration high " $\mathrm{H}$ ". The selection of a suitable corrosion protection system will be explained in section 4.3 .

Chapter 10 of EN 1090-4 [1] gives some basic requirements for choosing the appropriate corrosion protection system. The detailed requirements and an explanation of how to achieve the requirements are included in annex $\mathrm{E}$ of EN 1090-4. The manufacturer proves through an initial type testing (ITT) that a coating system meets these requirements.

In addition to the design requirements, the annex also specifies requirements for the production of corrosion protection systems. These are not relevant for users and are not dealt with further here.

\subsection{Suitability of a corrosion protection method}

EN 1090-4 [1] defines a number of corrosion protection systems that can be used for cold-formed structural steel members and sheeting. These corrosion protection systems include metal coatings such as continuously applied hot-dip coatings of zinc (Z), zinc-aluminium (ZA), aluminium-zinc (AZ) and zinc-magnesium-aluminium (ZM) according to EN 10346 [5] and hot-dip galvanizing according to EN 1461 [13]. Organic coatings such as coil coatings according to EN 10169 [5] and other liquid or powder coating systems are also included (where coil coating is always used in combination with continuously applied hot-dip metal coatings). Continuously applied hot-dip coatings and coil coatings are most important for the structural products according to EN 1090-4 [1]. 
As the suitability of an organic coating system is specified in EN 10169 without any reference to the application or the duration of protection, EN 1090-4 explicitly mentions the essential corrosion protection systems applicable for structural products in appendix E, Tables E.5 and E.6. The tables are only an overview of duplex coating systems and are not exhaustive. It is possible to use other corrosion protection systems for which proof of suitability has been provided.

The expected duration of protection is also given in Tables E.5 and E.6. This is an essential property that EN 10169 does not mention. The duration of protection is specified as the time until the loss of adhesion of the organic coating occurs. This does not mean that once the duration of protection of the organic coating has been reached, the construction product is not protected against corrosion and has thus reached the end of its working life. The metal coating still provides sufficient protection for a longer time, so measures can be taken (e.g. recoating or replacement of parts) before red rust appears and thus damage to the construction product occurs. EN 1090-4 does not provide for the metal coating to be included in the calculation of the protection duration. As the corrosion protection is essential for thingauged structural products, this ensures a higher level of safety against corrosion damage for the structural parts. It should be noted that a weathered coating with defects can absorb moisture, which accelerates the corrosion of the metal coating, especially if the surface cannot be washed by rain.

The durations of protection given in the tables are typical for the designated coating systems. The theoretically achievable duration of protection depends on many parameters such as the duration of protection between the primer and the top layer, ingredients such as colour pigments, etc. Therefore, the duration of protection of a coating system can be different to that given in the tables. Hence, the actual duration of protection of a specific coating should be specified by the coater.

According to section E.1, metal coatings without any organic coating may be used as well. Classification into corrosivity categories or duration of protection is not given in the standard. This is where the standard is inconsistent and ambiguous. Whereas on the one hand reference is made to the EN 14713 [14] series of standards for zinc, which permits zinc coatings in certain thicknesses for outdoor applications and any corrosivity categories, reference is also made here to the minimum requirements in Tables E.2 to E.4, which exclude outdoor use and partly indoor use. For the thickness of the metal coatings, EN 1090-4 [1] refers to EN 508-1 [8], where the minimum requirements are given for different countries (see section 2.2.2). This regulation only makes sense if Tables E. 2 to E.4 are used for metal coatings with the thicknesses according to EN 508-1. For other metal coating thicknesses it is necessary for the coater to state the duration of protection based on proof of suitability.
The duration of protection of metal coatings has been reached when red rust occurs. If the design working life of the structure is greater than the duration of protection, maintenance should take place before red rust occurs. EN 14713-1 [14] gives some information concerning this.

\subsection{Selection of a coating system}

The normative regulations for metal and coil coatings allow corrosion that is harmless from a constructional point of view and do not refer to aesthetic requirements, the use of the building, accessibility, visibility, maintenance intensity and sensitivity to mechanical stress or component function.

The atmospheric environment has a major influence on the selection of a suitable corrosion protection system. Tab. E.1 in EN 1090-4 [1] describes typical environmental atmospheres (outdoor for macroclimate and indoor for microclimate). By using this table, a suitable corrosion protection system can be selected for most cases. Nevertheless, this table is not a substitute for a closer look at the structure. Microclimate conditions should be checked on a case-by-case basis. Pollution due to infrastructure, industrial wastes, emissions from the building and adjacent buildings, thermal conditions, etc. are the decisive factors. However, the corrosivity on a surface also depends to a considerable extent on the cleaning regime. A dirty surface on which condensation often forms will be more exposed to corrosion than a clean, dry surface. It should also be mentioned that Tab. E.1 was adopted from EN ISO 12944-2 [3] and cannot always be used directly for coil coating according to EN 10169 [6]. For example, many coating systems assigned to corrosivity category C3 according to EN 1090-4 [1] are not suitable for loads from food processing plants or laundries, which are designated as atmosphere C3 in Tab. E.1. Therefore, for harsh microclimate conditions, the proper corrosion protection system must be determined in consultation with the coil coater. In addition to the environmental conditions, the function of the construction product and the installation situation have a great influence on the proper choice of the corrosion protection system. Different requirements are placed on accessible and non-accessible roofs. Aesthetic requirements, however, are generally influenced by the expectations of the building user. UV resistance and the desired or required robustness (resistance to, for instance, foot traffic) are other important factors in the selection of a suitable corrosion protection system. Therefore, depending on the type of use and visibility of the building component, it may be necessary to select higher resistances than would be required for corrosion protection against the atmospheric environment.

On the basis of the former German standard for trapezoidal sheets and liner trays, DIN 18807-1 [15], tables were compiled for EN 1090-4 [1], annex E, Tables E.2 to E.4, to determine the minimum requirement of the corrosion protection system for wall, roof and ceiling systems de- 
Tab. 4 Corrosivity categories/Duration of protection for roof systems - excerpt from Tab. E.3, EN 1090-4

\begin{tabular}{ll}
\hline \multicolumn{2}{l}{ Single skin, with thermal insulation top, non-ventilated } \\
\hline $\begin{array}{ll}\text { Weather side } \\
\text { (here: interior atmosphere) }\end{array}$ & Generally: \\
\hline $\begin{array}{l}\text { Non-weather side } \\
\text { (here: interior atmosphere) }\end{array}$ & Above dry, mainly closed rooms, (no condensation, no special loading): \\
\cline { 2 - 2 } & Z or ZM or ZA or AZ \\
\cline { 2 - 2 } & Generally: \\
& $\begin{array}{l}\text { Z or ZM or ZA or AZ and additional organic coating or AZ only } \\
\text { duration of protection high }\end{array}$ \\
\hline
\end{tabular}

(1): "Duration of protection high" means that a coating system shall be chosen according to the prevailing corrosivity category.

pending on the applications of the profiled sheets or members. Thus, depending on the exposure (exterior or interior application) and the type of construction (e.g. single-skin non-insulated, single-skin thermally insulated, double-skin ventilated), it is easy to select the corrosion protection system. The corrosivity of the environment is not considered in these tables and can lead to a higher corrosion protection requirement.

The standardization process, with many European countries involved, led to a solution that generalizes the choice of corrosion protection system to a large extent. In the published form it is a signpost to some degree, but not a real selection guide. It is difficult to create such a guide for all countries owing to the different approaches. For Germany, the IFBS association recently published such a guide [16], which gives more assistance than EN 1090-4 [1].

Tab. 4 shows an excerpt from Tab. E.3 for a single skin, top-side thermally insulated, non-ventilated construction. The designer can evaluate which corrosion protection requirements have to be followed depending on the side of the steel sheet and the accompanying atmospheric conditions.

\subsection{Galvanic corrosion}

Galvanic corrosion occurs when a metal is electrically coupled with another, nobler metal or conductive material while in contact with an electrolyte. The corrosion rate is higher than the particular corrosion rate of the metal as such. The less noble metal corrodes. The corrosion rate heavily depends on the electrode potential difference between the contact pairs and on the area ratio of the contact pairs. Galvanic corrosion is caused by unfavourable design or poor execution of the structure.

Section E.2.4 in EN 1090-4 [1] gives information on suitable design to prevent galvanic corrosion. Organically coated structural members are usually adequately protected against electrical conduction and thus against galvanic corrosion. From the electrochemical point of view, the coating of the nobler partner has a higher importance for protecting against galvanic corrosion. Areas of contact of different metals should be separated permanently by additional coatings or barrier layers where there is a possibility of corrosion due to contact between structural members and sheeting and fasteners made from different metals. Tab. E.7 gives information about combining different metal partners.

The galvanic corrosion depends very much on the area ratio of the contact partners. As the surface of the noble metal increases, so the risk of galvanic corrosion also increases. Therefore, materials for fasteners should always be the same as or nobler than the material of the structural members and sheeting being fastened.

Although galvanic corrosion can only occur when there is contact between two metals and an electrolyte is present, the components do not need be in direct contact. The transport of metal ions by rainwater is often a cause of galvanic corrosion. For example, if copper is used in the building and is exposed to the weathering (e.g. ground wire, moss removal systems), the copper ions propagating in the direction of the flow of the rainwater are sufficient to destroy zinc-based coatings within a very short time.

\subsection{Fasteners}

According to EN 1090-4, 5.7 [1], fasteners that are completely or partially exposed to weathering or similar moisture loads shall be made from austenitic stainless steel or aluminium. The term "partially exposed" does not mean brief exposure to weathering during installation. Alternatively, the standard permits to show by testing that the corrosion protection system of the exposed part of carbon steel fasteners corresponds to the corrosion protection of the parts to be connected. This does not apply to weldedon drill tips. Such proof can hardly be provided. In some European countries, e.g. Germany, only stainless steel fasteners are permitted under weathered conditions.

For fasteners that are not made from stainless steel, the corrosion protection of the fasteners should be adapted to the required corrosion protection of the parts to be connected by means of galvanizing and, if necessary, an organic coating. The requirements in EN ISO 4042 [17] must be considered. In the case of electrogalvanizing, the coating thickness shall be at least $8 \mu \mathrm{m}$. A thinner coating 
might be acceptable, provided specific verification of the durability is given for the intended use of the fasteners.

The requirement that the layer thickness for electrogalvanizing must be at least $8 \mu \mathrm{m}$ corresponds to the requirement in EN 1993-1-3 [18], annex B. However, it must be emphasized that this coating may only be used in corrosivity category $\mathrm{C} 1$ - thus only in dry interiors! For all other environmental conditions, the Eurocode also requires a minimum coating of $45 \mu \mathrm{m}$.

In any case, section 5.7 of EN 1090-4 [1] contains the wording "if not otherwise specified", which means that national regulations may supersede the regulations of the standard.

New coating system: zinc-magnesium-aluminium (ZM)

More than 10 years ago, European research on continuously hot-dip coated products led to the development of a new generation of protective metal coatings on steel which were based on ternary alloys of zinc, aluminium and magnesium. These products are obtained by immersing a prepared strip in a molten bath of zinc, aluminium and magnesium.

Thanks to improved corrosion resistance properties, the use of ZM-based coating systems is gradually increasing for many different applications (automotive, solar, industry, etc.), including building elements (structural or otherwise). These ZM metallic coatings can be used on their own or combined with an additional organic coil coating.

Since 2015 these new hot-dip coated steel flat products with a zinc-magnesium-aluminium alloy (ZM) are regulated in EN 10346 [5] alongside zinc (Z), zinc-aluminium (ZA) and aluminium-zinc (AZ) coatings. In this ZM products range, the composition of the material used in the hot-dip bath is a mix of aluminium and magnesium including aluminium in a range off 1.5 to $8 \%$, with minimum $0.2 \%$ magnesium, and the balance is zinc. The final coating density depends on the alloy composition. However, EN 10346 [5] does not regulate the corrosion resistance of the coatings. The corrosion protection provided by zinc-magnesium-aluminium (ZM) alloy has been introduced in the standards; since 2018 this has been regulated in Germany by DIN 55634-1 [19] and in Europe by EN 1090-4 [1]. These new ZM coatings are not yet included in the non-harmonized standard EN 508-1 [8], but will be included in the next revision.

The typical microstructure of a zinc-magnesium-aluminium alloy is made up of zinc grains surrounded by a lamellar structure consisting of binary, eventually tertiary, eutectics. The proportion of lamellar structure increases with the content of magnesium and aluminium in the alloy. In ZM alloys with a high aluminium content, aluminium dendrites can also be present. When exposed while the steel product is in use, this lamellar structure induces the formation of specific protective oxides, leading to increased corrosion protection.

In many scientific publications it is stated that ZM coatings provide a significantly higher (sometimes up to 18 times higher) durability compared with zinc with the same coating mass. These statements are only valid for the specified tested conditions. Such tests are performed to understand the corrosion mechanism of the coating and do not provide any values for durability under natural environmental conditions. However, it is generally accepted, and now reported in standards such as DIN 55634-1 [19] or EN 1090-4 [1], that a given ZM coating thickness has at least a corrosion resistance behaviour similar to a zinc (Z) coating twice as thick. The manufacturer shall verify this resistance of his individual ZM coating within the initial type testing. Any higher performance has to be proved. For this, the manufacturer has the option of applying for an ETA (European Technical Assessment) or, in some countries, a National Technical Approval.

In terms of applications, the ZM coatings are now used in many different fields (automotive, solar, electrical equipment, HVAC, general industry, etc.). They can be used indoors or outdoors, in the different corrosivity categories C defined in EN ISO 12944-2 [3]. The coating thickness has to be selected as a function of the corrosivity category $\mathrm{C}$ involved and the expected durability. The thickness of the steel substrate to be protected with the ZM coating layer also has to be considered for this selection.

\section{Conclusions}

The international standard series EN ISO 12944 contains the basics for corrosion protection systems on steel. However, the standard does not take account of thin-walled steel structures. For thin-walled steel structures, corrosion protection is regulated in a large number of standards. For self-supporting products, the corrosion protection is regulated in EN 14782 in combination with EN 508. EN 10169 considers coil-coated sheets with a focus on organic coating for all types of application. This standard became the state of the art for most applications with thin-walled structures. Since EN 10169 does not consider specific applications, it does not provide the designer with any assistance in the question of which corrosion protection to plan for the individual structural part. The introduction of EN 1090-4 has partially closed the gap for loadbearing structural parts. Even if this standard is incomplete with regard to the rules for corrosion protection and new coating systems and still contains errors, it offers a basis for the corrosion protection of loadbearing thin-walled elements.

\section{Acknowledgement}

Open Access funding enabled and organized by Projekt DEAL. 


\section{References}

[1] EN 1090-4 (2018) Execution of steel structures and aluminium structures - Part 4: Technical requirements for cold-formed structural steel elements and cold-formed structures for roof, ceiling, floor and wall applications.

[2] EN ISO 12944-1 (2017) Paints and varnishes - Corrosion protection of steel structures by protective paint systems Part 1: General introduction.

[3] EN ISO 12944-2 (2017) Paints and varnishes - Corrosion protection of steel structures by protective paint systems Part 2: Classification of environments.

[4] EN ISO 12944-5 (2019) Paints and varnishes - Corrosion protection of steel structures by protective paint systems Part 5: Protective paint systems.

[5] EN 10346 (2015) Continuously hot-dip coated steel flat products for cold forming - Technical delivery conditions.

[6] EN 10169:2010+A1:2012 (2012) Continuously organic coated (coil coated) steel flat products - Technical delivery conditions.

[7] EN 14782 (2006) Self-supporting metal sheet for roofing, external cladding and internal lining - Product specification and requirements.

[8] EN 508-1 (2014) Roofing and cladding products from metal sheet - Specification for self-supporting of steel, aluminium or stainless steel sheet - Part 1: Steel.

[9] EN 10204 (2004) Metallic products - Types of inspection documents.

[10] EN 1990 (2002) + A1:2005 + A1:2005/AC (2010) Eurocode: Basis of structural design.

\section{Authors}

Dipl.-Ing. Alexander Britner (corresponding author) alexander.britner@kit.edu

Karlsruhe Institute of Technology

Versuchsanstalt für Stahl, Holz und Steine

Kaiserstr. 12

76131 Karlsruhe, Germany

Ing. Corinne Dieu

corinne.dieu@arcelormittal.com

ArcelorMittal Europe - Flat Products

Rue Verte Voie, 49

4000 Sclessin, Belgium

Dr.-Ing. Ralf Podleschny

info@ifbs.eu

IFBS - Internationaler Verband für den Metallleichtbau

Europark Fichtenhain A 13a

47807 Krefeld, Germany
[11] EN 1993-1-1:2005 + AC (2009) Eurocode 3: Design of steel structures - Part 1-1: General rules and rules for buildings.

[12] EN 1993-1-4:2006 + A1 (2015) Eurocode 3: Design of steel structures - Part 1-4: General rules - Supplementary rules for stainless steels.

[13] EN ISO 1461 (2009) Hot dip galvanized coatings on fabricated iron and steel articles - Specifications and test meth ods.

[14] EN ISO 14713-1 (2017) Zinc coatings - Guidelines and recommendations for the protection against corrosion of iron and steel in structures - Part 1: General principles of design and corrosion resistance.

[15] DIN 18807-1:1987-06 (1987) Trapezprofile im Hochbau; Stahltrapezprofile; Allgemeine Anforderungen, Ermittlung der Tragfähigkeitswerte durch Berechnung. Berlin: Beuth.

[16] IFBS (2021) IFBS-Fachregeln des Metallleichtbaus: Grundlagen. Krefeld: IFBS

[17] EN ISO 4042 (2018) Fasteners - Electroplated coating systems.

[18] EN 1993-1-3:2006 + AC:2009 (2009), Eurocode 3: Design of steel structures - Part 1-3: General rules - Supplementary rules for cold-formed members and sheeting.

[19] DIN 55634-1:2018-03 (2018) Beschichtungsstoffe und Überzüge - Korrosionsschutz von tragenden dünnwandigen Bauteilen aus Stahl - Teil 1: Anforderungen und Prüfverfahren. Berlin: Beuth.

\section{How to Cite this Paper}

Britner, A.; Dieu, C.; Podleschny, R. (2021) Corrosion protection for cold-formed structural steel elements. Steel Construction. https://doi.org/10.1002/stco.202100002

This paper has been peer reviewed. Submitted: 28. January 2021 accepted: 17. April 2021 